\title{
A CLASSIFICATION OF BRAID TYPES FOR PERIODIC ORBITS OF DIFFEOMORPHISMS OF SURFACES OF GENUS ONE WITH TOPOLOGICAL ENTROPY ZERO
}

\author{
J. Guaschi, J. Llibke AND R.S. MAckay
}

Abstract

We classify the braid types that can occur for finite unions of periodic orbits of diffeomorphisms of surfaces of genus one with zero topological entropy.

\section{Introduction}

In this paper we classify the braid types that can occur for finite unions of periodic orbits of diffeomorphisms of surfaces of genus one with zero topological entropy. This extends the analysis from the case of genus zero [LM1]. The case of most interest to us is diffeomorphisms of the torus, isotopic to the identity. This is relevant to the behaviour of three coupled oscillators, for example. A good picture of their dynamics is developing [KMG], [LM2], [MZ], [H2], [F], [BGKM]. We hope that our results will help solve the intriguing problem of understanding the boundary of zero topological entropy in the space of $C^{1}$ diffeomorphisms of the torus.

We begin by establishing some notation and recalling the definition of braid type. Let $f: X \rightarrow X$ be a diffeomorphism of an oriented manifold $X$. Write $h(f)$ for the topological entropy of $f$. We write $o-p, o-r$ for orientationpreserving and reversing, respectively. Given two diffeomorphisms $f: X \rightarrow$ $X, g: Y \rightarrow Y$ of oriented manifolds, we write $f \simeq g$ if there exists an $o-p$ conjugacy between them.

Let $M$ be a surface, i.e. a compact connected oriented 2-manifold. Let $f: M \rightarrow M$ be a diffeomorphism of $M$ and let $\mathcal{P}$ be a finite union of periodic orbits for $f$. Then we define $f_{\mathcal{P}}: M_{\mathcal{P}} \rightarrow M_{\mathcal{P}}$ by removing $\mathcal{P}$ from $M$ and recompactifying by replacing the points of $\mathcal{P}$ by circles on which $f \mathcal{p}$ is the projective action of $D f[B]$. 
Given two diffeomorphisms $f, g: X \rightarrow X$ of a surface $X$ and finite unions $\mathcal{P}, \mathcal{Q}$ of periodic orbits for $f, g$ respectively, we say the pairs $(\mathcal{P}, f)$ and $(\mathcal{Q}, g)$ have the same braid type if there exists an $o-p$ homeomorphism $k: X_{\mathcal{P}} \rightarrow X_{\mathcal{Q}}$ such that $k f p k^{-1}$ is isotopic to $g_{\mathcal{Q}}$. The equivalence class of $(\mathcal{P}, f)$, denoted $[\mathcal{P}, f]$, is called its broid type.

To specify the braid type of a pair $(\mathcal{P}, f)$ we will use Nielsen-Thurston theory to select a simplest representative of the equivalence class. We refer the reader to [LM1] for the necessary information about Nielsen-Thurston theory of classification of surface homeomorphisms up to isotopy, and the definitions of the classes of diffeomorphisms which we call disc trees, reversing disc trees and reversing annulus trees.

The plan of the paper is as follows. In Section 2, we classify finite order homeomorphisms of the torus, which is an important preliminary result. Then in Section 3, we use Nielsen-Thurston theory to isotope $f_{p}$ to a standard form, and analyse the possibilities. Our main result is Theorem 4 , but because it is rather long to state and requires notions introduced in Section 2, we leave its statement until Section 3. In Section 4 we rederive results of [H1], [H2], and [LM2] as corollaries of those of Section 3.

We thank the referee for a careful reading. The first two authors were partially supported by a SERC studentship and a DGICYT grant respectively.

\section{Classification of finite order homeomorphisms of the torus}

In this section, we give a classification of finite order homeomorphisms of the torus up to $o-p$ conjugacy. Let $T_{x}, x \in \mathbb{F}^{2}$, be translation by $x$ on $\mathbb{R}^{2}$, i.e. $T_{x}(y)=y+x, y \in \mathbb{R}^{2}$. Let $R_{\omega}, \omega \in \mathbb{R} / 2 \pi \mathbb{Z}$ be rotation about 0 on $\mathbb{R}^{2}$ by angle $\omega$. Let $r$ be the reflection

$$
r\left\{\begin{array}{l}
x^{\prime}=x \\
y^{\prime}=-y
\end{array}\right.
$$

on $\mathbb{R}^{2}$. Define $\Gamma_{0}$ to be the group generated by $T_{(1,0)}$ and $T_{(0,1)}, \Gamma_{\Delta}$ that generated by $T_{(1,0)}$ and $T\left(\frac{1}{2}, \frac{\sqrt{3}}{2}\right)$. If $f: \mathbb{R}^{2} \rightarrow \mathbb{P}^{2}$ commutes with the action of a group $\Gamma$ on $\mathbb{R}^{2}$, we define $f / \Gamma: \mathbb{R}^{2} / \Gamma \rightarrow \mathbb{R}^{2} / \Gamma$ by identifying points of the same orbit under $\Gamma$. For $q \in \mathbb{N}$, let $\tilde{\mathbb{Z}}_{q}$ be the quotient of $\mathbb{Z}$ under the equivalence relation generated by the relations $p \sim p+q, p \sim-p$.

Theorem 1. If $f: \bar{\nabla}^{2} \rightarrow \bar{\nabla}^{2}$ is a homeomorphism of finite order, then it is $o-p$ conjugate to one of the following: 
(a) $T_{(p, 0) / q} / \Gamma_{\square}$ for some $q \in \mathbb{N}, p \in \tilde{\mathbb{Z}}_{q}$, with $p, q$ having no common factor (order $q$ ).

(b) $R_{\omega} / \Gamma_{\square}, \omega= \pm \pi / 2, \pi$, or $R_{\omega} / \Gamma_{\Delta}, \omega= \pm \pi / 3, \pm 2 \pi / 3$ (orders $4,2,6,3$ respectively) (see Figure 1).

(c) $r \circ T_{(p, 0) / q} / \Gamma_{\square}$, for some $q \in \mathbb{N}, p \in \tilde{\mathbb{Z}}_{q}$, with $p$, $q$ having no common factor (order $q$ if $q$ is even, order $2 q$ if $q$ is odd).

(d) $r \circ R_{\pi / 2} / \Gamma_{\square}$ (order 2) (see Figure 2).

No two of the above are $o-p$ conjugate.

The proof of Theorem 1 reduces to the classification of isometries of tori, a well-studied problem (e.g. see [NS]). However, we have not found a compact treatment in the literature, nor one which considers the question of which isometries are equivalent up to $o-p$ conjugacy. So we give a derivation in the Appendix.

For case (b) of Theorem 1, the numbers of periodic orbits of smaller period than the order are given by the following theorem.

Theorem 2 ([E]). The rotation of order 2 has 4 fixed points. The rotations of order 3 have 3 fixed points. The rotations of order 4 have 2 fixed points and one orbit of period 2. The rotations of order 6 have one fixed point, one orbit of period 2, and one orbit of period 3. (See Figure 1).

\section{Results}

We firstly recall some definitions and theorems that we will need. We then go on to state and prove our results.

Let $f: M \rightarrow M$ be a homeomorphism of a surface of genus one, then its completion $g: \mathbb{T}^{2} \rightarrow \mathbb{T}^{2}$, is defincd by considering $M$ to be $\bar{\pi}^{2}$ minus a disjoint union of equal size discs $D_{i}$, and extending $\left.g\right|_{\partial D_{i}}$ radially into $D_{i}[E]$. If $h(f)=0$, then $h(g)=0$. We say a simple closed curve on $M$ is rotational if it is homotopically non-trivial on $\mathbb{J}^{2}$ after filling in the holes.

We recall the following two results.

Theorem A ([LM1]). Let $f: X \rightarrow X$ be a homeomorphism of a surface of genus zero. If $f$ is $o-p$, it has either a fixed point or an invariant boundary component. If $f$ is $o-r$ it has either a fxed point or an invariant boundary component, or an orbit of period 2 or a boundary component of period 2.

So in both the $o-p$ and $o-r$ cases, if $\mathcal{P}$ is a finite union of periodic orbits for $f$, and $f_{\mathcal{P}}$ does not have a boundary component of period I $(o-p)$ or period 1 or $2(o-r)$, we can always append a fixed point or period 2 orbit to $\mathcal{P}$ in order to achieve this. 
Theorem B ([LM1]). Let $f: X \rightarrow X$ be a diffeomorphism of a surface of genus zero, with $h(f)=0$, and let $\mathcal{P}$ be a finite union of periodic orbits.

(1) If $\mathcal{P}$ contains a fixed point or $f$ has an invariant boundary component, then

(a) If $f$ is $o-p,[\mathcal{P}, f]$ has a representative which is a disc tree.

(b) If $f$ is $o-r,[\mathcal{P}, f]$ has a representative which is a reversing disc tree.

(2) If $f$ is $o-r$, and $\mathcal{P}$ contains no fixed point and $f$ has no invariant boundary component, but either $\mathcal{P}$ has a point of period 2 or $f$ has a boundary component of period 2 , then $[\mathcal{P}, f]$ has a representative which is a reversing annulus tree.

Let $f: M \rightarrow M$ be a diffeomorphism of a surface of genus one with $h(f)=0$, and let $\mathcal{P}$ be a finite union of periodic orbits for $f$. Let $F$ be a Thurston canonical form for $f_{p}$. Then from Thurston's classification of surface homeomorphisms, $F$ is either reducible, or of finite ordcr. First we consider the case that $F$ has a rotational reducing curve.

Theorem 3. Suppose $f: M \rightarrow M$ is a diffemorphism of a surface of genus one with $h(f)=0$, and $\mathcal{P}$ is a finite union of periodic orbits for $f$. Let $F$ be a Thurston canonical form for $f_{p}$, such that $F$ has a rotational reducing curve $\Gamma$, of period $p$. Remove the tubular neighbourhood of $\Gamma$ and its images, to obtain a disjoint union of punctured annuli $A_{i}$. Then

(i) If all $A_{i}$ have period $p$, then $[\mathcal{P}, f]$ has a representative which is $p$ annuli, joined by generalised twists, permuted like a rotation, and if $f$ is $o-p$ or $p$ is even there exists a disc tree $d: X^{\prime} \rightarrow X^{\prime}$ such that $F^{p} \mid A_{i} \simeq d$ or if $f$ is $o-r$ and $p$ is odd there exists a reversing disc tree $d^{\prime}: X^{\prime} \rightarrow X^{\prime}$ such that $F^{p} \mid A_{i} \simeq d^{\prime}$.

(ii) Suppose some $A_{i}$ has period $q \neq p$ (so $q=p / 2$ ), then $p=2$, and there are two such annuli $A_{1}, A_{2}$. If $f$ is $o-p,[\mathcal{P}, f]$ has a representative which is two annuli, joined by generalised twists, and there exist disc trees $D_{i}: X^{\prime} \rightarrow X^{\prime}, i=1,2$ such that $F \mid A_{i} \simeq D_{i}$. If $f$ is $o-r,[\mathcal{P}, f]$ has a representative which is two annuli, joined by generalised twists, and for each of $A_{1}, A_{2}$ there exist reversing disc trees $D^{\prime}: X^{\prime} \rightarrow X^{\prime}$ such that $F \mid A_{i} \simeq D^{\prime}$ or reversing annulus trees $S_{i}: X \rightarrow X$, such that $F \mid A_{i} \simeq S_{i}$, according as $F \mid A_{i}$ has an invariant boundary component or fixed point, or not.

Proof: Suppose $\Gamma$ has period $p$. Define $\Lambda=\left\{F^{k} \Gamma: 0 \leq k<p\right\}$. Then $F$ permutes the elements of $\Lambda$. If we remove the tubular neighbourhood of $\Gamma$ and its images, we obtain a disjoint union of annuli $A_{i}$ with holes, which are 
permuted. Then the $A_{i}$ either have period $p$, or if $p$ is even, some of the $A_{i}$ may have period $p / 2$ (i.e. the two boundaries of $A_{i}$ in $\Lambda$ are interchanged by $\left.F^{p / 2}\right)$. This gives two cases:

(i) If the $A_{i}$ have period $p, F$ permutes the $A_{i}$, and there are two subcases:

(a) If $f$ is $o-p$, or if $f$ is $o-r$ and $p$ is even, then $F^{p} \mid A_{i}$ is $o-p$, so by Theorem $B$, there exists a disc tree $d: X^{\prime} \rightarrow X^{\prime}$ such that $F^{p} \mid A_{i} \simeq d$.

(b) If $f$ is $o-r$ and $p$ is odd, then $F^{p} \mid A_{i}$ is $o-r$, so by Theorem $\mathrm{B}$, there exists a reversing disc tree $d^{\prime}: X^{\prime} \rightarrow X^{\prime}$ such that $F^{p} \mid A_{i} \simeq d^{\prime}$.

(ii) Suppose $p$ is even, and there exists some $A_{i}$ (without loss of generality $i=0$ ) such that $A_{i}$ has period $p / 2$. Put $q=p / 2$. Then we claim that $q=1$.

To prove the claim, suppose $A_{0}$ has period $q>1$. Writc $A_{k}=F^{k}\left(A_{0}\right), 1 \leq$ $k<q$. Consider the situation on the torus; then each $A_{k}$ has two boundary components $\Gamma_{k}^{(1)}, \Gamma_{k}^{(2)} \in \Lambda$, say, for $0 \leq k<q$. Then for $j \neq k, \Gamma_{j}^{(i)} \neq \Gamma_{k}^{(l)}$, i $l=1,2$, i.e. no two distinct $A_{k}$ have a boundary component in common. For suppose $\Gamma_{j}^{(i)}=\Gamma_{k}^{(l)}$ for some $i, l \in\{1,2\}, j \neq k$ (see Figure $3(a)$ ). Then since $F^{q}$ interchanges $\Gamma_{k}^{(1)}$ and $\Gamma_{k}^{(2)}$ for each $k$, then $F^{q}\left(\Gamma_{j}^{(l)}\right)=\Gamma_{j}^{(i+1)}=F^{q}\left(\Gamma_{k}^{(i)}\right)=$ $\Gamma_{k}^{(l+1)}$; hence there are only two distinct elements of $\Lambda$, so $q=1$, a contradiction.

Since the $A_{k}$ have no boundary components in common, there exist precisely $q$ tubular regions $B_{1}, \ldots, B_{q}$, such that each $B_{i}$ lies between two $A_{k}$, i.e. each $B_{i}$ has two distinct elements of $\Lambda$ as its (rotational) boundary components. Consider one such element of $\left\{B_{i}\right\}_{i=1}^{q}, B$, say. Then without loss of generality, it lies between $A_{0}$ and $A_{k}$, for some $k$, and has boundary components $\Gamma_{0}^{(1)}, \Gamma_{k}^{(1)} \in \Lambda$ (see Figure $\left.3(\mathrm{~b})\right) . B$ is invariant under $F^{2 q}$. Since $\Gamma$ has period $2 q, B$ must have period either $q$ or $2 q$. Suppose it has period $q$, then $F^{q}$ interchanges its boundary components, so $\Gamma_{k}^{(1)}=F^{q}\left(\Gamma_{0}^{(1)}\right)$. But $F^{q}$ interchanges the boundary components of $A_{0}$ so $F^{q}\left(\Gamma_{0}^{(1)}\right)=\Gamma_{0}^{(2)}$, therefore $A_{0}$ and $A_{k}$ have a boundary component in common, which we have shown does not occur. Hence $B$ has period $2 q$.

However $F^{k}\left(A_{0}\right)=A_{k}$, for $k<q$, and considcring $F^{k+q}\left(A_{0}\right)=A_{k}$, if necessary, then $F^{k}\left(\Gamma_{0}^{(1)}\right)=\Gamma_{k}^{(1)}$, and $F^{k}\left(\Gamma_{0}^{(2)}\right)=\Gamma_{k}^{(2)}$. But the elements of $\Lambda$ are permuted like a rotation or rotation with reflection by $F$, since $f$ is a diffeomorphism, hence $F^{k}\left(\Gamma_{k}^{(1)}\right)=\Gamma_{0}^{(1)}$, therefore $F^{k}(B)=B$, a contradiction, as $B$ has period $2 q$. This proves the claim.

So $q=1$, and $\Gamma$ has period 2, with $\Lambda=\{\Gamma, F(\Gamma)\}$. If we remove the tubular neighbourhoods of $\Gamma$ and $F(\Gamma)$, we obtain two disjoint annuli $A_{0}$ and $A_{1}$. Then there are two subcases to consider: 
(a) If $f$ is $o-p$, then $F \mid A_{i}(i=0,1)$ is $o-p$, so by Theorem $B,[P, f]$ has a representative which is two annuli joined by generalised twists, such that $F \mid A_{i}$ is a disc tree.

(b) If $f$ is $r-o$, then $F \mid A_{i}(i=0,1)$ is $o-r$, so by Theorem $B,[\mathcal{P}, f]$ has a representative which is two annuli joined by generalised twists, such that $F \mid A_{i}$ is a reversing disc tree or a reversing annulus tree, according as $f$ has an invariant boundary component or $\mathcal{P}$ contains a fixed point in $A_{i}$, or not.

This completes the proof of Theorem 3.

Next we consider the case where there is no rotational reducing curve. Either $F$ is of finite order, or has a non-rotational reducing curve $C$, in which case we may remove the decomposition components (of genus zero) of $C$ and its images from $M$. So in both cases, we can find a unique decomposition component $S$ of genus one, such that $F \mid S$ is of finite order. Let $G: \mathbb{J}^{2} \rightarrow \mathbb{J}^{2}$ be the completion of $F \mid S$. Then $G$ is of finite order, so is $o-p$ conjugate to one of the cases of Theorem 1.

This leads to the following theorem, which is our main result:

Theorem 4. Suppose $F: M \rightarrow M$ is a Thurston canonical form for a homeomorphism of a surface of genus one, with unique decomposition component $S$ of genus one. Let $G: \mathbb{T}^{2} \rightarrow \mathbb{T}^{2}$ be the completion of $F \mid S$. Then one of the following is true:

(a) $G \simeq T_{(p, 0) / q} / \Gamma_{\square}$, and all boundary components of $S$ have period $q$. Further, for each orbit $\partial_{1}, \ldots, \partial_{q}$ of boundary components of $S$ not in $\partial M$, there exists a disc tree $d: X^{\prime} \rightarrow X^{\prime}$ such that the component $X_{i}$ of $M \backslash S$ inside $\partial_{i}$ is homeomorphic to $X^{\prime}$, and $F^{q} \mid X_{i} \simeq d$.

(b) $G \simeq R_{\omega} / \Gamma_{\square}, \omega= \pm \pi / 2, \pi$ (order $k=4,2$ ), or $G \simeq R_{\omega} / \Gamma_{\Delta}, \omega=$ $\pm \pi / 3, \pm 2 \pi / 3$ (order $k=6,3$ ). If $k=2$, then all boundary components of $S$ have period 1 or 2 , with at most 4 of period 1. If $k=3$, then all boundary components of $S$ hove period 1 or 3 , with at mosi 3 of period 1. If $k=4$, then all boundary components of $S$ have period 1, 2 or 4 , with at most 2 of period 1 , and 1 orbit of period 2 . If $k=6$, then all boundary components of $S$ have period 1, 2, 3 or 6 with at most 1 of period 1, 1 orbit of period 2, and 1 orbit of period 9 . In case, for each orbit of boundary components of period $p, \partial_{1}, \ldots, \partial_{p}$, of $S$, not in $\partial M$, there exists a disc tree $d: X^{\prime} \rightarrow X^{\prime}$ such that the component $X_{i}$ of $M \backslash S$ inside $\partial_{i}$ is homeomorphic to $X^{\prime}$, and $F^{p} \mid X_{i} \simeq d$.

(c) (i) $G \simeq\left(r \circ T_{(p, 0) / q}\right) / \Gamma_{0}$. If $q$ is even, all boundary components have period $q$. If $q$ is odd, all boundary components have period $q$ or $2 q$.

(ii) $G \simeq\left(r \circ R_{\pi / 2}\right) / \Gamma_{\square}$. All boundary components of $S$ have period either 1 or 2 . 
In both parts of case (c), for each orbit of boundary components of period $p, \partial_{1}, \ldots, \partial_{p}$, of $S$ not in $\partial M$, there exisis a disc tree $d: X^{\prime} \rightarrow X^{\prime}$ such that the component $X_{i}$ of $M \backslash S$ inside $\partial_{i}$ is homeomorphic to $X^{\prime}$ and $F^{p} \mid X_{i} \simeq d$ if $p$ is even, or there exists a reversing disc tree $d^{\prime}: X^{\prime} \rightarrow X^{\prime}$ such that the component $X_{i}^{\prime}$ of $M \backslash S$ inside $\partial_{i}$ is homeomorphic to $X^{\prime}$ and $F^{p} \mid X_{i}^{\prime} \simeq d^{\prime}$ if $p$ is odd.

Proof: We apply Theorem 1 to the completion $G: \mathbb{J}^{2} \rightarrow \mathbb{T}^{2}$ of $F \mid S$.

Case (a) $G \simeq T_{(p, 0) / q} / \Gamma_{\square}$, so all points of $\mathbb{T}^{2}$ have period $q$ under $G$. Hence all boundary components of $S$ have period $q$, and if we consider the orbits $\partial_{1}, \ldots, \partial_{q}$ of those not in $\partial M$, the corresponding decomposition components $X_{i}$ of $M \backslash S$ inside $\partial_{i}$ are of genus zero, and we may apply Theorem $B$, so there exists a disc tree $d: X^{\prime} \rightarrow X^{\prime}$ such that $X_{i}$ is homeomorphic to $X^{\prime}$, and $F^{q} \mid X_{i} \simeq d$.

Case (b) $G \simeq R_{\omega} / \Gamma_{\square}, \omega= \pm \pi / 2, \pi: k=4,2$, or $G \simeq R_{\omega} / \Gamma_{\Delta}, \omega=$ $\pm \pi / 3, \pm 2 \pi / 3: k=6,3$.

The statements about the orbits of boundary components are an immediate consequence of applying Theorem 2 to the completion $G: \mathbb{\nabla}^{2} \rightarrow \mathbb{T}^{2}$. As in case (a), since $G$ is $o-p$, we obtain disc trees in each orbit of boundary components of $S$ not in $\partial M$.

Case (c) (i) $G \simeq\left(r \circ T_{(p, 0) / q}\right) / \Gamma_{\square}$.

From the proof of Theorem 1 , the boundary components of $S$ have period $q$ if $q$ is even, or they have period $q$ or $2 q$ if $q$ is odd.

Case (c) (ii) $G \simeq\left(r \circ R_{\pi / 2}\right) / \Gamma_{\square}$.

Again from the proof of Theorem 1, the boundary components of $S$ have period 1 or 2 .

In both parts of case (c), each component of $\nabla^{2} \backslash S$ inside a boundary component not in $\partial M$ of even period $r$ contains a disc tree since $F^{r}$ is $Q-p$, whilst those of odd period $r^{\prime}$ contain a reversing disc tree since $F^{r^{\prime}}$ is $o-r$, by Theorem B. 


\section{Two Corollaries}

As a corollary of the results of Section 3 we obtain the genus one case of [H1]:

Theorem 5. Let $f: M \rightarrow M$ be an $o-r$ diffeomorphism of a surface $M$ of genus one. If $f$ has periodic orbits, or orbits of boundary components, with 9 distinct odd periods, then $h(f)>0$.

To derive this from the above, we require the genus zcro result of $[\mathrm{BF}],[\mathrm{H} 1]$, also derived by [LM1]:

Theorem C. Let $f: X \rightarrow X$ be an o- $r$ diffeomorphism of a surface of genus zero. If $f$ has periodic orbits or orbits of boundary components with two distinct odd periods, then $h(f)>0$.

Proof of Theorem 5: Let $f: M \rightarrow M$ be an $o-r$ diffeomorphism of a surface $M$ of genus one. Let $\mathcal{P}$ be the union of three orbits of distinct odd period. Let $F$ be a Thurston canonical form for $f_{\mathcal{P}}$. Suppose $h(f)=0$. Then there are two cases:

Case (a): Suppose there is a rotational reducing curve, $\Gamma$, say, with period $p$. Remove its annular neighbourhood and its images, then we obtain a disjoint union of annuli $A_{i}$. From Theorem 3, there are two possibilities. In the first case, the $A_{i}$ have period $p$, so the $A_{i}$ are permuted by $F$, and thus $p$ divides the order of each periodic orbit, hence $p$ is odd. So if we consider any $A_{i}, F^{p} \mid A_{i}$ is $o-r$, since $f$ is $o-\tau$. $A_{i}$ must contain boundary components corresponding to the three orbits of distinct odd period. So by Theorem $C, h(f)>0$, a contradiction.

The other possibility is when $p=2$, and there are two invariant annuli $A_{0}, A_{1}$. Then one of $A_{0}, A_{1}$ must contain boundary components corresponding to at least two of the three orbits, and since $F \mid A_{i}(i=0,1)$ is $o-r$, Theorem $\mathrm{C}$ implies that $h(f)>0$, a contradiction.

Case (b): Suppose there is no rotational reducing curve. Then there exists a unique decomposition component $S$ of genus one. Let $G: \mathbb{T}^{2} \rightarrow \mathbb{T}^{2}$ be the completion of $F \mid S$. Then we are in Case (c) of Theorem 4. If $G \simeq\left(r \circ T_{(p, 0) / q}\right) / \Gamma_{\square}$, all boundary components of $S$ have period $q$ or $2 q$. If $G \simeq\left(r \circ R_{\pi / 2}\right) / \Gamma_{\square}$, then all boundary components of $S$ have period 1 or 2 . In particular, all boundary components of $S$ of odd period have the same period $p$, and the remaining boundary components corresponding to $\mathcal{P}$ must lie within decomposition components $X_{i}$ of genus zero whose outer boundaries have period $p$. Since $F^{p} \mid X_{i}$ is $o-r$, and at least one of the $X_{i}$ contains boundary components of odd order, 
not $p$, corresponding to the orbits of $\mathcal{P}$, then Thcorem $\mathrm{C}$ implics that $h(f)>0$, a contradiction.

As a second corollary wc will derive a result of [LM2], [H2] for diffeomorphisms of the torus isotopic to the identity. We refer the reader to [LM2] for definitions of lifts, rotation vectors, etc. We recall that for a continuous map $f: \mathbb{T}^{2} \rightarrow \mathbb{J}^{2}$ with lift $\tilde{f}: \mathbb{R}^{2} \rightarrow \mathbb{B}^{2}$, if $\Gamma$ is the group of integer translations $\gamma_{m}: x \mapsto x+m, x \in \mathbb{R}^{2}, m \in \mathbb{Z}^{2}$, and if $f$ is homotopic to the identity, then

$$
\tilde{f} \gamma=\gamma \tilde{f} \text { for all } \gamma \in \Gamma \text {. }
$$

Theorem 6. Let $f: \mathbb{T}^{2} \rightarrow \mathbb{T}^{2}$ be a homeomorphism of the torus isotopic to the identity, and suppose $h(f)=0$. Then all rotation vectors associated with the periodic orbits of $f$ are collinear.

Proof: Suppose that $f$ has a finite union of periodic orbits, with associated distinct rotation vectors $p_{i} / q_{i}, i=1, \ldots, N$. Then from [LM2], since $f$ is homotopic to the identity, for each $i \in\{1, \ldots, N\}$ there exists a periodic orbit. $Q_{2}$ of primitive rotation type $\left(p_{i}, q_{i}\right)$. Let. $\mathcal{P}=\bigcup_{i=1}^{N} Q_{i}$, and let $F$ be a Thurston canonical form for $f_{p}$.

Suppose $F$ is of finite order, then using $(*)$ and Theorem 1 , we see that $F \simeq \tau_{(p, 0) / q} / \Gamma_{\square}$, for some $q \in \mathbb{N}, p \in \tilde{\mathbb{Z}}_{q}$, so all points of $\mathbb{T}^{2}$ are periodic with period $q$ and rotation vector $(p, 0) / q$.

The remaining possibility is that $F$ is reducible (with finite order components, though we shall not need this). There are no non-rotational reducing curves for $F$. For suppose $\Gamma$ were such a curve, then it must surround at least two holes, but they must come from the same orbit, so the rotation type of that orbit cannot be primitive, which is a contradiction.

Suppose there is a rotational reducing curve $\Gamma$ for $F$. Let $G: \mathbb{T}^{2} \rightarrow \nabla^{2}$ be the completion of $F$, and let $\tilde{G}: \mathbb{R}^{2} \rightarrow \mathbb{R}^{2}$ be a lift of $G$. Let $m \in \mathbb{Z}^{2} \backslash\{0\}$ be the homotopy type of $\Gamma$, and $q$ be its period. Then $\Gamma$ lifts to an infinite set of curves $\tilde{\Gamma}$, each invariant under $T_{m}$, which partition the plane into infinite strips $\vec{S}$ each within a bounded distance of some straight line of direction $m$. Furthermore, there exists $p \in \mathbb{Z}^{2}$ such that $F^{a} T_{p} \hat{\Gamma}=\tilde{\Gamma}$, and since $F$ is invertible the same holds for the strips $\tilde{S}$. Hence the rotation set of $F$, and in particular the rotation vectors of the chosen periodic orbits, are contained in the straight line $\{p / q+t m: t \in \mathbb{R}\}$.

This completes the proof.

\section{Appendix: Proof of Theorem 1}

To prove Theorem 1, we require the following: 
Theorem $7[\mathrm{E}]$. If $f$ is a finite order homeomorphism of a compact orientable manifold $M$, then there exists a Riemannian metric $\mathcal{R}$ of constant curvature on $M$ such that $f$ is a diffeomorphism preserving $\mathcal{R}$.

Let $\mathcal{E}$ be the Euclidean metric on $\mathbb{R}^{n}$, and let $E^{n}$ be the group of isometries of $\left(\mathbb{R}^{n}, \mathcal{E}\right)$. It consists of all transformations $y \mapsto A y+x$, with $x \in \mathbb{R}^{n}, A \in O(n)$. In the case $n=2, E^{2}$ is generated by $T_{x}, R_{\omega}$ and $r$, where $x \in \mathbb{R}^{2}, \omega \in \mathbb{R} / 2 \pi \mathbb{Z}$.

Theorem 8 (Killing, Hopf, see [W]). Let $\left(M^{n}, \mathcal{R}\right)$ be a Riemannian manifold of dimension $n \geq 2$ with metric $\mathcal{R}$. Then $\left(M^{n}, \mathcal{R}\right)$ is complete, connected and of constant curvature $K=0$ if and only if it is isometric to a quotient $\left(\mathbb{R}^{n}, \mathcal{E}\right) / \Gamma$, where $\Gamma$ is a subgroup of $E^{n}$ which acts freely and properly discontinuously.

Theorem 9 ([S]). Suppose $R_{\omega} \in S O(2), R_{\omega} \neq \mathrm{Id}$, commutes with $\Gamma$ on $\mathbb{R}^{2}$, where $\Gamma$ is a subgroup of $E^{2}$ with two generators, and which acts freely and properly discontinuously. Then $\omega= \pm \pi / 3, \pm \pi / 2, \pm 2 \pi / 3$, or $\pi$, and $\Gamma$ is conjugate in the group generated by $S O(2)$ and isotropic scale changes to $\Gamma_{\square}$ if $\omega= \pm \pi / 2$ or $\pi$, or $\Gamma_{\Delta}$ if $\omega= \pm \pi / 3$ or $\pm 2 \pi / 3$.

Proof of Theorem 1: Suppose $f$ is a finite order homeomorphism of $\sigma^{2}$. Theorem 7 implies that there exists a Riemannian metric $\mathcal{R}$ of constant curvature on $\mathbb{T}^{2}$, such that $f$ is a diffeomorphism and preserves $\mathcal{R}$. By the Gauss-Bonnet formula (c.g. [DC]), the curvature is zero. By Theorem $8,\left(\pi^{2}, \mathcal{R}\right.$ ) is isometric to $\left(\mathbb{R}^{2}, \mathcal{E}\right) / \Gamma$, for some $\Gamma<E^{2}$. Since $\sigma^{2}$ is compact, $\Gamma$ must be a group generated by two translations $T_{y_{1}}, T_{y_{2}}$, with $y_{1}, y_{2}$ linearly independent, and without loss of generality, $y_{1}$ is along the $x$-axis (by rotating coordinates). To find the finite order isometries $f:\left(\mathbb{T}^{2}, \mathcal{R}\right) \circlearrowleft$, it suffices to find all the isometries $\tilde{f}:\left(\mathbb{R}^{2}, \mathcal{E}\right) \nrightarrow$, such that $\tilde{f} \Gamma=\Gamma \tilde{f}$, and $\tilde{f}^{m} \in \Gamma$, for some $m$. If $f$ is $o-r$, then $r \circ f$ is $o-p$ and satisfies the same conditions. So let us take $f$ to be $o-p$.

If $\tilde{f}$ has no fixed point, then it is a translation $T_{x}, x \in \mathbb{R}^{2}$. If $\tilde{f}^{m} \in \Gamma$ then $x$ is a rational combination of $y_{1}$ and $y_{2}$. Hence $f \simeq T_{p / q} / \Gamma_{\square}$, for some $p \in \mathbb{Z}^{2}, q \in$ $\mathbb{N}$. However, many of these are $o-p$ conjugate. Let $\frac{p}{q}=\frac{\left(p_{1}, p_{2}\right)}{q}$, which we assume is in lowest terms. Suppose $p_{1}$ and $p_{2}$ have no common factors. Consider the orbit of $T_{p / q} / \Gamma_{\square}$ under $S L(2, \mathbb{Z})$. Then for $A=\left[\begin{array}{ll}a & b \\ c & d\end{array}\right] \in S L(2, \mathbb{Z})$,

$$
\left[\begin{array}{ll}
a & b \\
c & d
\end{array}\right]\left[\begin{array}{l}
p_{1} / q \\
p_{2} / q
\end{array}\right]=\frac{\left(a p_{1}+b p_{2}, c p_{1}+d p_{2}\right)}{q}
$$

and $\frac{a p_{1}+b p_{2}}{q}$ and $\frac{c p_{1}+d p_{2}}{q}$ have no common factors. For suppose $a p_{1}+b p_{2}=$ $k n_{1}, c p_{1}+d p_{2}=k n_{2}$, for some integers $n_{1}, n_{2}, k \neq \pm 1$. Then 


$$
\left[\begin{array}{l}
p_{1} \\
p_{2}
\end{array}\right]=k A^{-1}\left[\begin{array}{l}
n_{1} \\
n_{2}
\end{array}\right]
$$

but we assumed that $p_{1}$ and $p_{2}$ were coprime, a contradiction. Now $p_{1}$ and $p_{2}$ are coprime if and only if there exist integers $a, b$ such that $a p_{1}+b p_{2}=1$. Let $c=-p_{2}$ and $d=p_{1}$, then

$$
\left[\begin{array}{ll}
a & b \\
c & d
\end{array}\right]\left[\begin{array}{l}
p_{1} \\
p_{2}
\end{array}\right]=\left[\begin{array}{l}
1 \\
0
\end{array}\right] \text { and } A=\left[\begin{array}{ll}
a & b \\
c & d
\end{array}\right] \in S L(2, \mathbb{Z})
$$

Conversely given coprime integers $a, c$ we can find integers $b, d$ satisfying $a d-$ $b c=1$, and

$$
\left[\begin{array}{ll}
a & b \\
c & d
\end{array}\right]\left[\begin{array}{l}
1 \\
0
\end{array}\right]=\left[\begin{array}{l}
a \\
c
\end{array}\right] \text { and } A=\left[\begin{array}{ll}
a & b \\
c & d
\end{array}\right] \in S L(2, \mathbb{Z})
$$

Suppose $p_{1}$ and $p_{2}$ are not coprime but have highest common factor hcf $\left(p_{1}, p_{2}\right)=k$. Then $\frac{\left(p_{1}, p_{2}\right)}{q}=k \frac{\left(m_{1}, n\right)}{q}$, where $m$ and $n$ are coprime. Hence the orbit of $T_{p / q} / \Gamma_{\square}$ under $S L(2, \mathbb{Z})$ is all $T_{p^{\prime}} / q^{\prime} / \Gamma_{\square}$, with $q^{\prime}=q$ and hef $\left(p_{1}^{\prime}, p_{2}^{\prime}\right)=$ hef $\left(p_{1}, p_{2}\right)$, where $p^{\prime}=\left(p_{1}^{\prime}, p_{2}^{\prime}\right)$.

Thus every element in this orbit is a translation by $\frac{k}{q}(a, b)$, with $k=$ hef $\left(p_{1}, p_{2}\right)$, and hef $(a, b)=1$. Thus we may choose a representative $T_{(p, 0) / q} / \Gamma_{\square}$, with $p \in \tilde{\mathbb{Z}}_{q}$, and $p, q$ having no common factors.

If $\tilde{f}$ has a fixed point, we may assume by making a translation if necessary that $O$ is fixed. So $\tilde{f}(x)=A x$, for some $A \in S O(2)$. The case of $\tilde{f}=\mathrm{Id}$ is included in part (a). Otherwise, by Theorem $9, \Gamma$ is conjugate to $\Gamma_{\square}$ or $\Gamma_{\Delta}$, and $\omega= \pm \pi / 2, \pi$ in the case $\Gamma_{\square}, \pm 2 \pi / 3, \pm \pi / 3$ in the case $\Gamma_{\Delta}$.

Thus when $f$ is $o-p$, it is $o-p$ conjugate to one of the cases given in parts (a) and (b). It can be seen that no two of these cases are $o-p$ conjugate.

If $f$ is $o-r$, then it is $o-p$ conjugate to the composition of $r$ with one of the cases in parts (a) and (b). However, some of these are $o-p$ conjugate to each other, so they are not distinct cases. There are two classes to consider.

(i) $f \simeq g=\left(r \circ T_{(p, 0) / q}\right) / \Gamma_{\square}$ (Case (c)). On $\pi^{2}$, there are two curves invariant under $g$; they are $y=0$ and $y=\frac{1}{2}$. Under $g$, the $x$-coordinate has period $q$, and the $y$-coordinate has period $I$ if $y=0$ or $\frac{1}{2}$, and period 2 otherwise. So if $q$ is even all points in $\mathbb{\nabla}^{2}$ have period $q$. If $q$ is odd, all points in $\mathbb{T}^{2}$ have period $q$ or $2 q$, according as they lie on an invariant curve or not. The invariant curves have rotation number $p / q \in \tilde{\sigma}^{l}$, where $\tilde{j}^{1}$ stands for the quotient of $\mathbb{R}$ under 
the equivalence relation generated by $x \sim x+1, x \sim-x$. The rotation number is an invariant of $o-p$ conjugacy. Hence the cases in part (c) are distinct.

(ii) $f \simeq g=\left(r \circ R_{\omega}\right) / \Gamma_{\square}, \omega=\pi, \pm \pi / 2$, or $f \simeq g=\left(r \circ R_{\omega}\right) / \Gamma_{\Delta}, \omega=$ $\pm \pi / 3, \pm 2 \pi / 3$. Without loss of generality (by conjugation by $R_{-\omega}$ ), we take $\omega$ to be positive.

If $\omega=\pi$, then on $\mathbb{R}^{2}$ this corresponds to perpendicular reflection in $x=0$, so by a change of coordinates, $f \simeq r$, which is already included in Case (c).

If $\omega=\pi / 2$ (Case (d)), then on $\mathbb{R}^{2}$ this corresponds to perpendicular reflection in the curve $x+y=0$. It is the only invariant curve, and is composed of fixed points.

If $\omega=\pi / 3$, then on $\mathbb{R}^{2}$ this corresponds to perpendicular reflection in $x+$ $\sqrt{3} y=0$. On $\nabla^{2}$, there is one curve fixed pointwise by $g$, and $g$ is a reflection in it.

If $\omega=2 \pi / 3$, then on $\mathbb{P}^{2}$ this corresponds to perpendicular refection in $x+\frac{1}{\sqrt{3}} y=0$. On $\mathbb{T}^{2}, x+\frac{1}{\sqrt{3}} y=0$ is the only curve fixed pointwise by $g$, and $g$ is a reflection in it.

Let $h_{1}=r \circ R_{\pi / 2} / \Gamma_{\square}, h_{2}=r \circ R_{2 \pi / 3} / \Gamma_{\Delta}$. Then $h_{1}$ and $h_{2}$ are $o-p$ conjugate. To see this, we need to find an $o-p$ homeomorphism $k: \mathbb{R}^{2} / \Gamma_{\square} \rightarrow \mathbb{R}^{2} / \Gamma_{\Delta}$ such that $k h_{1}=h_{2} k$, or equivalently an $o-p$ homeomorphism $K: \mathbb{R}^{2} \rightarrow \mathbb{R}^{2}$ such that $K H_{1}=H_{2} K$, where $h_{1}=H_{1} / \Gamma_{\square}$ and $h_{2}=H_{2} / \Gamma_{\Delta}$, and also such that for each $\gamma_{\square} \in \Gamma_{\square}$ there exists a $\gamma_{\Delta} \in \Gamma_{\Delta}$ such that $K \gamma_{\square}=\gamma_{\Delta} K$ and vice versa. This is satisfed by

$$
K=\left[\begin{array}{cc}
1 & \frac{1}{2} \\
0 & \frac{\sqrt{3}}{2}
\end{array}\right]
$$

(with respect to the basis vectors of $\Gamma_{0}$ ), which is a shear taking the generators of $\Gamma_{\square}$ to $\Gamma_{\Delta}$. Hence $h_{1}$ and $h_{2}$ are $o-p$ conjugate. Similarly the case $r \circ R_{\pi / 3} / \Gamma_{\Delta}$ is $o-p$ conjugate to $h_{1}$, by taking

$$
K=\left[\begin{array}{cc}
1 & \frac{1}{2} \\
0 & \frac{\sqrt{3}}{2}
\end{array}\right] .
$$

The cases $h_{1}$ and $r$ are distinct because the case $r$ has two invariant curves, whilst the case $h_{1}$ bas only invariant curve. 
Figures

(a)

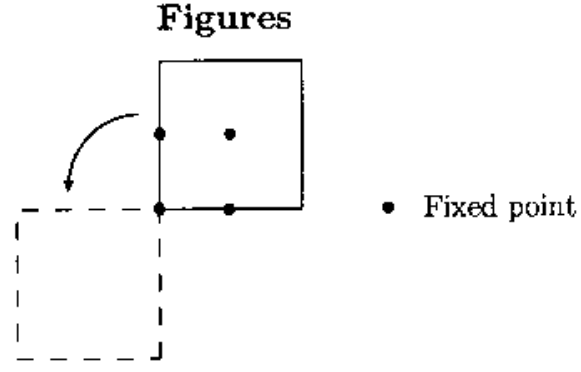

(b)
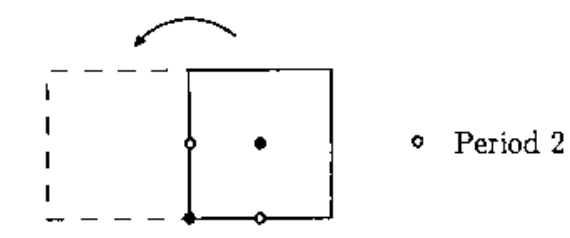

(c)

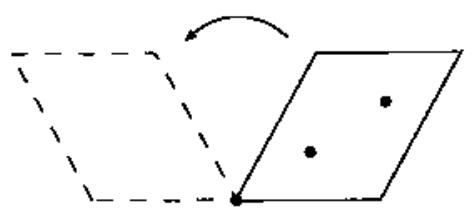

(d)

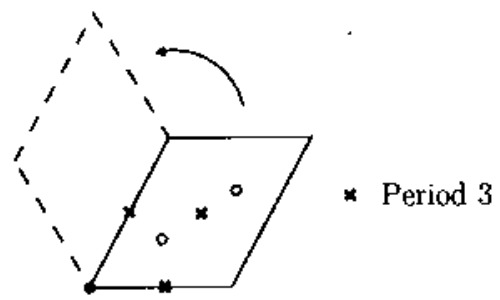

Figure 1: Finite order $o-p$ diffeomorphisms of the torus with a fixed point:
(a) $R_{\pi} / \Gamma_{\square}$
(b) $R_{\frac{\pi}{2}} / \Gamma_{\square}$
(c) $R_{\frac{2 \pi}{3}} / \Gamma_{\Delta}$
(d) $R_{\frac{\pi}{3}} / \Gamma_{\Delta}$ 


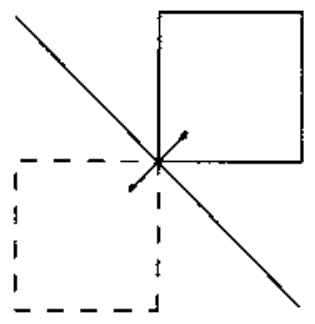

Figure 2: The $o-r$ diffeomorphism $r \circ R_{\frac{\pi}{2}} / \Gamma_{\square}$.

(a)

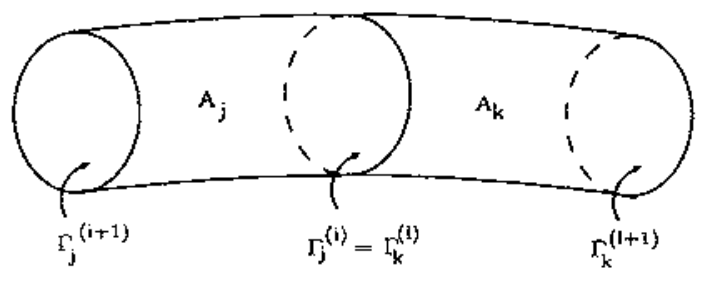

(b)

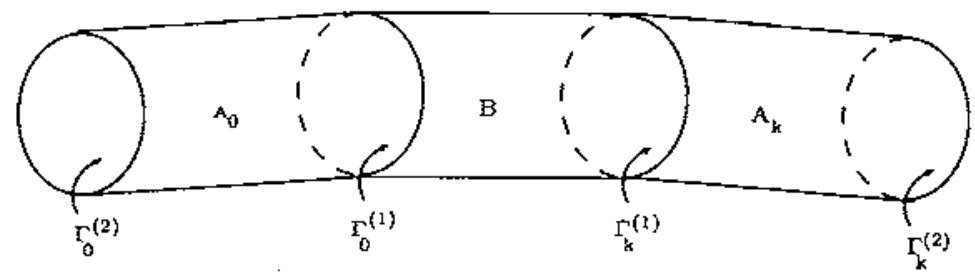

Figure 3: Proof of Theorem 6: (a) no two distinct $A_{k}$ have a boundary component in common, (b) a tubular region $B$. 


\section{References}

[BGKM] C. Baesens, J. Guckenhelmer, S. Kim and R.S. MaCKay, Three coupled oscillators: Mode locking, global bifurcations and toroidal chaos, Physica D. 49 (1991), 387-475.

(B) R. Bowen, "Entropy and the fundamental group," Springer Lecture Notes in Math. 668, 1978, pp. 21-29.

[BF] P. BLANChaRd AND J. FRANKS, The dynamical complexity of orientation-reversing homeomorphisms of surfaces, Invent. Math. 62 (1980), 333-339.

[DC] M.P. Do CARMo, "Differential Geometry of curves and surfaces," Prentice Hall, 1976.

[E] D.B.A. Epstein, Pointwise periodic homeomorphisms, Proc. London Math. Soc. 42 (1981), 415-460.

[Ei] S. EIL.ENBERG, Sur les transformations periodiques de la surface de sphere, Fund. Math. 22 (1934), 28-41.

[F] J. FRANKS, Realizing rotation vectors for torus homeomorphisms, Trans. Amer. Math. Soc. 311 (1989), 107-115.

[H1] M. HANDEL, The entropy of orientation reversing homeomorphisms of surfaces, Topology 21 (1982), 291-296.

[H2] M. Handel, Periodic point free homeomorphisms of the torus, Proc. Amer. Math. Soc. 107 (1989), 511-516.

[Ke] B. KeREKJARTO, Uber die periodischen transformationen der Kreisscheibe und der Kugelflache, Math. Ann. 80 (1919), 36-38.

[KMG] S. KIM, R.S. MACKaY A.ND J. GUCKENHEmer, Resonance regions for torus maps, Nonlinearity 2 (1989), 391-404.

[LM1] J. LLIBRE AND R.S. MACKAY, A classification of braid types for diffeornorphisms of surfaces of genus zero with topological entropy zero, $J$. London Math. Soc. 42 (1990), 562 576.

[LM2] J. LLIBRE AND R.S. MACKAY, Rotation vectors and entropy for homeomorphisms of the torus isotopic to the identity, Ergod. Th. Dyn. Sys. 11 (1991), 115-128.

[MZ] M. Misiurewicz and K. Ziemlan, Rotation sets for maps of tori, J. London Math. Soc. 40 (1989), 490-506. 
[NS] V.V. Nikulin AND I.R. Shafarevich, "Geometries and groups," Springer, 1987.

[S] R.L.E. SChWARzenberger, " $N$ dimensional crystallography," Pitman research notes in Mathematics 41, 1980.

[W] J.A. Wolf, "Spaces of constant curvature," McGraw-Hill, 1967.

J. Guaschi: Nonlinear Systems Laboratory

Mathernatics Institute

University of Warwick

Coventry CVA 7AL

ENGLAND

J. Llibre: Departament de Matemàtiques

Facultat de Ciencies

Universitat Autonoma de Barcelona

08193 Bellaterra (Barcelona)

SPAIN

R.S. Mackay: Nonlinear Systems Laboratory

Mathematics Institute

University of Warwick

Coventry CV4 7AL

ENGLAND

Primera versió rebuda el 23 d'Agost de 1990 ,

darrera versió rebuda el 2 de Setembre de 1991 